\title{
Role of non-symmetry-breaking order parameters in determining the martensitic energy barrier: The bcc-to-9R transformation
}

\author{
R. J. Gooding \\ Department of Physics, Queen's University, Kingston, Ontario, Canada K7L 3N6 \\ Y. Y. Ye, ${ }^{*}$ C. T. Chan, K. M. Ho, and B. N. Harmon \\ Ames Laboratory, Department of Physics, Iowa State University, Ames, Iowa 50011
}

(Received 15 November 1990)

\begin{abstract}
Distortions that do not affect the symmetry of the final product phase obtained in a martensitic transition, so-called non-symmetry-breaking order parameters, but that do affect the energy barrier encountered during homogeneous nucleation, are considered. For the bcc-to- $9 R$ transition undergone by $\mathrm{Na}$, first-principles total-energy calculations verify the importance of these distortions in that a $40 \%$ reduction is obtained when the non-symmetry-breaking order parameters are allowed to alter the transformation path defined only by the symmetry-breaking order parameters.
\end{abstract}

As a function of temperature or pressure, numerous crystals undergo martensitic transformations from one structure to another. ${ }^{1}$ These transformations range from weakly to strongly first order, and involve the cooperative (diffusionless) displacement of atoms, so that the paths followed by the atoms can usually be described by a manageable number of generalized coordinates, or order parameters. One conventional approach to studying the instability leading to such transformations is through Ginzburg-Landau-Wilson Hamiltonians. ${ }^{2}$ These phenomenological expressions involve expanding the free energy in a limited set of the parent phase's degrees of freedom (the order parameters). The set of order parameters chosen for a particular study is usually the minimum necessary to describe the symmetry-breaking displacements taking the parent (high-temperature) phase to the product (low-temperature) phase. In this Brief Report, we point out that selecting only the minimal set of order parameters (based on the change of symmetry between the two phases) may lead to unrealistically high estimates for the thermal activation energy. The point is that to properly describe the physics of these first-order phase transitions, it is not only necessary to establish the conditions when the two phases have a degenerate Gibbs free energy, but also the energy barrier along the configuration path separating the two phases must be correctly determined. ${ }^{3}$ As the system moves along this path, certain structural relaxations can lower the barrier height, and it is precisely in describing this phenomenon that it becomes important to introduce non-symmetrybreaking order parameters. The bcc-to- $9 R$ transformation $^{4}$ in $\mathrm{Na}$ offers an interesting example of such a case.

Many metals undergo a martensitic transformation from a high-temperature bcc phase to a low-temperature close-packed structure. For the alkali metals, the transformation occurs at rather low temperatures $\sim 75 \mathrm{~K}$ for $\mathrm{Li}$ and $\sim 35 \mathrm{~K}$ for $\mathrm{Na}$ ). In these metals, as for many bcc metals, the transverse-phonon modes belonging to the $\Sigma_{4}$ branch along the [110] direction are low in energy (small restoring forces), and it is the displacements associated with the phonon eigenvectors of this branch, together with their anharmonic coupling to elastic strain, that accounts for the transformation path. (The phonon energies do not go to zero as there are no soft-mode transitions in these systems. However, these weak restoring forces do signal the possibility of an incipient instability.) To describe the bcc-to- $9 R$ transformation, Gooding and Krumhansl ${ }^{5}$ took note of these weak restoring forces and thus were motivated to consider a Ginzburg-LandauWilson effective free energy which included displacements of the $\left(\frac{1}{3}, \frac{1}{3}, 0\right) \Sigma_{4}$ phonon and strains associated with the $\frac{1}{2}\left(c_{11}-c_{12}\right)$ elastic constant, the latter of which corresponds to the $q \rightarrow 0$ sound waves of this same phonon branch. These strains can be written as

$$
e_{2}=\frac{1}{\sqrt{2}}\left(e_{x x}-e_{y y}\right)
$$

and

$$
e_{3}=\frac{1}{\sqrt{6}}\left(e_{x x}+e_{y y}-2 e_{z z}\right)
$$

while the phonon displacements are written as

$$
\Psi=A \widehat{e}_{[1 \overline{1} 0]} \sin [(\mathbf{q} \cdot \mathbf{r})+\phi] .
$$

A linear combination of these three order parameters represents precisely the symmetry-breaking displacements that connect the bcc and $9 R$ structures. Starting from the expression for the free-energy density,

$$
\begin{aligned}
\widetilde{F}= & \frac{1}{2}\left(c_{11}-c_{12}\right)\left(e_{2}^{2}+e_{3}^{2}\right) \\
& +\frac{1}{3} c_{333} e_{3}\left(e_{3}^{2}-3 e_{2}^{2}\right)+\frac{1}{4} c_{222}\left(e_{2}^{2}+e_{3}^{2}\right)^{2} \\
& +\frac{1}{2} r_{0}|\Psi|^{2}+\frac{1}{4} u_{0}|\Psi|^{4}+\frac{1}{6} v_{0}|\Psi|^{6} \\
& +\frac{1}{6} v_{1}\left[\Psi^{6}+\left(\Psi^{*}\right)^{6}\right]+\frac{1}{8} w_{0}|\Psi|^{8}+\gamma e_{2}\left[\Psi^{3}+\left(\Psi^{*}\right)^{3}\right],
\end{aligned}
$$

one then deduces the following expression for the 
effective free-energy density along the proposed transformation path in terms of the amplitude $A$ appearing in Eq. (3):

$$
\begin{aligned}
\widetilde{F}= & \frac{1}{2} r_{0} A^{2}+\frac{1}{4} u_{0} A^{4} \\
& +\frac{1}{6}\left(v_{0}+2 v_{1}-\frac{12 \gamma^{2}}{c_{11}-c_{12}}\right) A^{6}+\frac{1}{8} w_{0} A^{8} .
\end{aligned}
$$

In this equation, the development of $e_{2}$ and $e_{3}$ is implicitly included when $A \neq 0$; restrictions on the value of $\phi$ in Eq. (3) are also implied. ${ }^{5}$

We now introduce the notion of a non-symmetrybreaking order parameter. By this we mean that we include possible distortions of the product phase, in this case the $9 R$ structure, as well as intermediate crystalline structures encountered along the path connecting the parent and product phases; the new distortions are not relevant in obtaining a product phase with a given symmetry. For the bcc-to-9R transformation, the nonsymmetry-breaking order parameters considered here are

$$
e_{1}=\frac{1}{\sqrt{3}}\left(e_{x x}+e_{y y}+e_{z z}\right)
$$

and

$$
e_{6}=e_{x y} .
$$

Thus, additional terms appear in the free-energy expansion:

$$
\widetilde{F}^{\prime}=\frac{1}{2}\left(c_{11}+2 c_{12}\right) e_{1}^{2}+\frac{1}{2} c_{44} e_{6}^{2}+\gamma_{1} e_{1}|\Psi|^{2}+\gamma_{2} e_{6}|\Psi|^{2} .
$$

The $e_{1}$ corresponds to a homogeneous volume change, and $e_{6}$ provides another degree of freedom which allows the $c / a$ ratio to be varied independently. ${ }^{6}$ These new terms lead to a reduction of the fourth-order term in Eq. (5), viz.,

$$
u_{0} \rightarrow u_{0}-\frac{1}{2} \frac{\gamma_{1}^{2}}{c_{11}+c_{12}}-\frac{1}{2} \frac{\gamma_{2}^{2}}{c_{44}},
$$

and thus to a reduction of the energy barrier. To be specific, the alteration of the fourth-order term is more important to the energy barrier than is the destabilizing sixth-order term (see Ref. 5) simply because a lower power of the amplitude $A$ is involved.

These considerations can be concretely illustrated by first-principles total-energy calculations ${ }^{7}$ which were recently completed in the study of the bcc-to- $9 R$ martensitic transformation for $\mathrm{Na}$. These calculations considered the three symmetry-breaking order parameters and one non-symmetry-breaking order parameter, the $c / a$ ratio. The order parameters used are illustrated in Fig. 1 and include a tilt, $\Delta$, away from the [110] bcc direction; a Bain strain, $\theta$; the $c / a$ ratio; and a phonon displacement, $\delta$. These order parameters can be used to represent the same displacements as $e_{2}, e_{3}, e_{6}$, and $A$ and were discussed by $\mathrm{Nagasawa}^{8}$ and Kelly ${ }^{9}$ in proposing a set of displacements between the two phases. In Fig. 2, we show the variation of these four order parameters along the minimum energy path from the bcc to the $9 R$ structure. In Fig. 3, the energy variation along this path is shown, and the energy barrier obtained has a value of 4.3 $\mathrm{K} /$ atom. Figure 2 is very revealing, especially with respect to the role played by the non-symmetry-breaking $c / a$ order parameter. Since the values of $c / a$ for the bcc and the $9 R$ structures are quite similar (these are the only values available from experiment), one might be led to ignore such terms in the free energy; however, the calculations show that the $c / a$ varies considerably along the path and is certainly coupled to the other parameters. By constraining the $c / a$ ratio at the bcc value and finding a new minimum-energy path, we arrive at a barrier height of $6.2 \mathrm{~K} /$ atom (an increase of $\sim 40 \%$ ). Simply put, by allowing the (110) planes to be pulled apart (increased $c / a$ ), the planes may slide over one another more easily, and then $A \neq 0$ may be developed with less energy required. This is clearly displayed in Figs. 2(a) and 2(b).

We have also investigated the effect of volume change on the barrier height, and found it to be small (see Fig. 4). Given that the ratio $\left(c_{11}+2 c_{12}\right) / c_{44} \sim 4$ in $\mathrm{Na},{ }^{10}$ this very small dependence of the energy barrier on $e_{1}$ is not unexpected. However, the calculations do show a slight dependence of the minimum barrier on this distortion
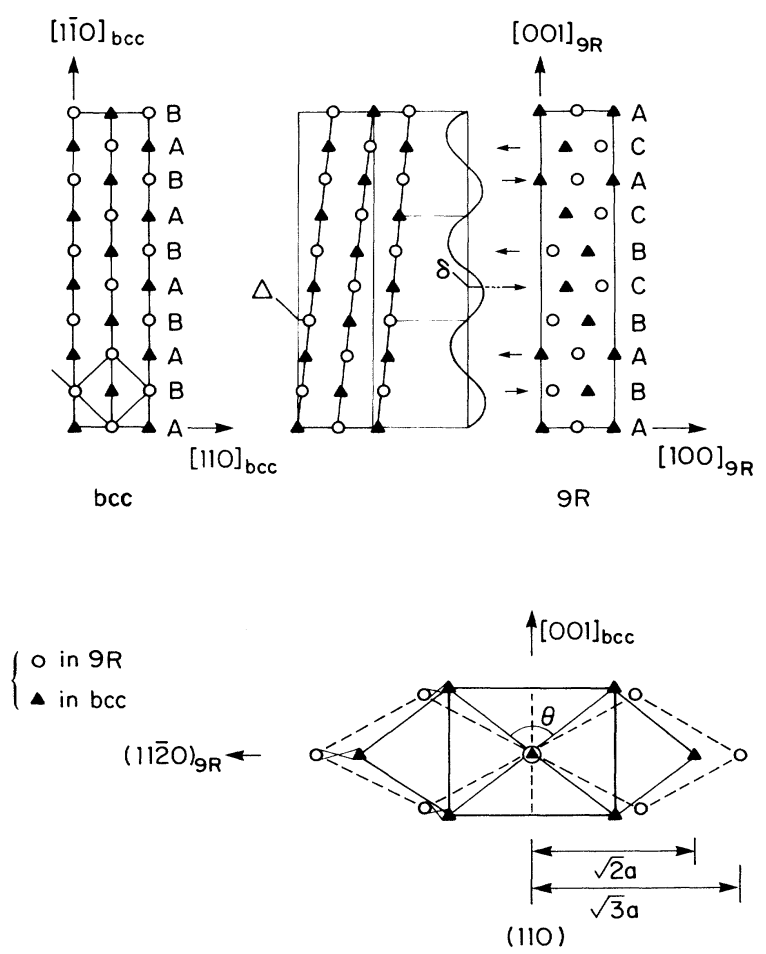

FIG. 1. Transformation between the bcc and the $9 R$ structure can be accomplished by (1) transforming the (110) basal planes to close packed-that is, changing $\theta$ from $109.47^{\circ}$ to $120^{\circ}$-and (2) obtaining the $\cdots / A B A B C B C A C / \cdots$ stacking sequence of close-packed planes by tilting the bcc [110] axis $(\Delta)$ and imposing atomic displacements according to the polarization vectors of a transverse $\frac{1}{3}[110]$ phonon $(\delta)$. (Reproduced from Ref. 7 with permission of the publisher.) 

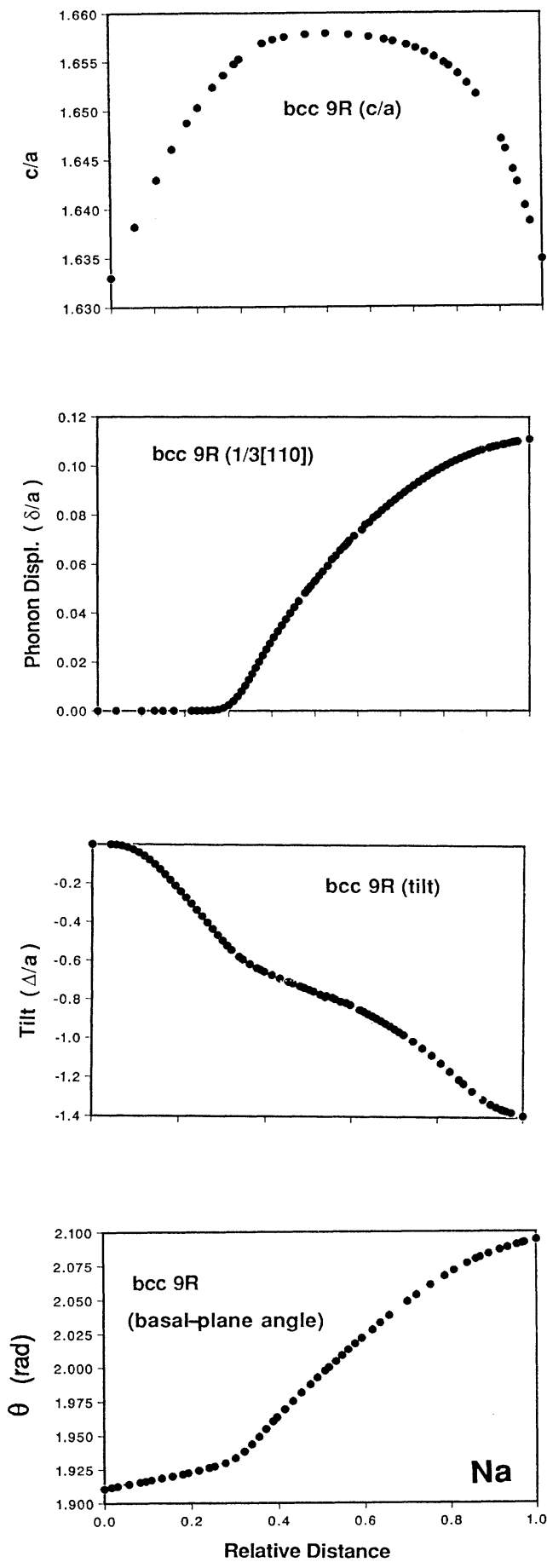

along minimum-energy path between the bcc and $9 R$ structures

FIG. 2. Variation of the individual order parameters along the minimum-energy path in the configuration space for the bcc-to- $9 R$ transformation in Na. $\Delta$ denotes the tilt away from the bcc[110] axis; $\delta$ denotes the displacement of the $\frac{1}{3}[110]$ phonon. (Reproduced from Ref. 7 with permission of the publishers.)

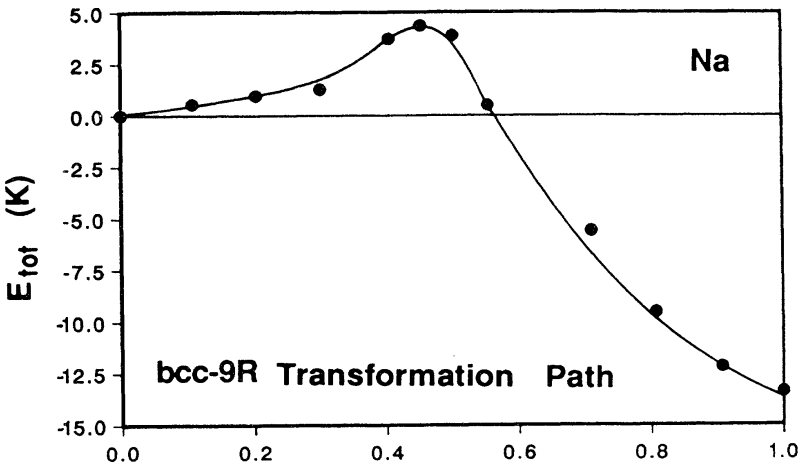

FIG. 3. Total-energy change along the "minimum-energy path" in the configuration space of transformation coordinates for the bcc-to- $9 R$ transition of $\mathrm{Na}$. The energy scale is in degrees Kelvin $(1 \mathrm{meV}=11.6 \mathrm{~K})$. (Reproduced from Ref. 7 with permission of the publishers.)

(viz., Fig. 4 is not symmetric about $V=V_{0}$ ), in agreement with the asymmetry implied by Eq. (8). There are some martensitic transformations which are strongly first order and the volume changes can be quite large $(\sim 21 \%$ in $\mathrm{Sn}){ }^{11}$ In those cases one would expect, of course, that $e_{1}$ is an important non-symmetry-breaking order parameter that would have to be included to properly ascertain both the final product phase, as well as the correct value of the energy barrier to homogeneous nucleation.

In summary, phenomenological energy expansions and parameter-free total-energy calculations both illustrate the necessity of considering displacements important in obtaining the thermal activation energy in a martensitic transition, even though these sample displacements are not relevant in determining the symmetry of the final product phase.

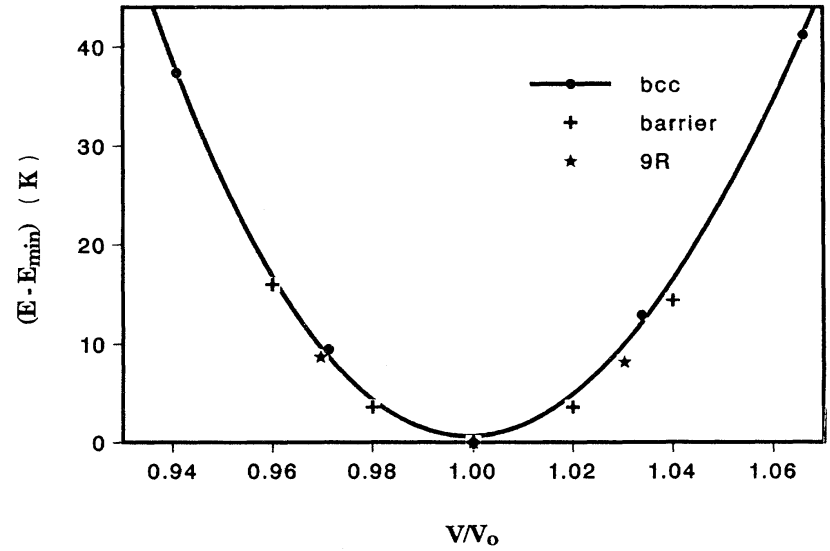

FIG. 4. Total crystalline energy as a function of volume for the structure defined by the bcc and $9 R$ phases, as well as by the atomic positions at the barrier peak. The energies are given relative to the minimum energies for these three structures, all of which occur at the same $V_{0}$, within the calculational precision. 
One of us (R.J.G.) acknowledges support from the Natural Sciences and Engineering Research Council of Canada. Ames Laboratory is operated for the U. S. Department of Energy by Iowa State University under Contract
No. W-7405-ENG-82. This work is supported by the Director of Energy Research, Office of Basic Energy Sciences.
*Permanent address: Physics Department, Wuhan University, Wuhan, People's Republic of China.

${ }^{1}$ Z. Nishiyana, Martensitic Transformations (Academic, New York, 1978).

${ }^{2}$ L. D. Landau and E. M. Lifshitz, Statistical Physics (AddisonWesley, Reading, MA, 1958); J.-C. Tolédano and P. Toledano, The Landau Theory of Phase Transitions (World Scientific, Singapore, 1987).

3J. S. Langer, Ann. Phys. (N.Y.) 54, 258 (1969); 65, 53 (1971); Physica 73, 61 (1974).

${ }^{4}$ R. Berliner, O. Fajen, H. G. Smith, and R. J. Hitterman, Bull. Am. Phys. Soc. 33, 615 (1988).
${ }^{5}$ R. J. Gooding and J. A. Krumhansl, Phys. Rev. B 38, 1695 (1988).

${ }^{6} \mathrm{~A}$ formal theory for the Landau expansion can be found in $\mathrm{I}$. Folkins and M. B. Walker, Phys. Rev. B 40, 255 (1989).

${ }^{7}$ Y.-Y. Ye, C. T. Chan, K. M. Ho, and B. N. Harmon, Int. J. Supercomputer Appl. 4, 111 (1990).

${ }^{8}$ A. Nagasawa, J. Phys. Soc. Jpn. 40, 1021 (1976).

${ }^{9}$ M. J. Kelly, J. Phys. F 9, 1921 (1979).

${ }^{10}$ P. Ho and A. L. Ruoff, J. Phys. Chem. Solids 29, 2101 (1968).

${ }^{11}$ D. L. Price and J. M. Rowe, Solid State Commun. 7, 1433 (1969). 